\title{
Dynamique hydrologique et évolution morpho sédimentaire du lac Ahémé au Sud-Ouest du Bénin
}

\author{
Sylvestre Yantikoua \\ Laboratoire de Géologie, Mines et Environnement, Département des \\ Sciences de la Terre, Université d'Abomey-Calavi \\ (LGME/DST/FAST/UAC). Ecole Doctoral Sciences de la Vie et de la Terre, \\ Université d'Abomey-Calavi (ED-SVT/FAST/UAC) \\ Raoul Laïbi \\ Rodrigue Adechina \\ Christophe Kaki \\ Laboratoire de Géologie, Mines et Environnement, \\ Département des Sciences de la Terre, Université d'Abomey-Calavi \\ (LGME/DST/FAST/UAC) \\ Daouda Mama \\ Laboratoire d'Hydrologie Appliquée, l'Institut Nationale de l'Eau, \\ Université d'Abomey-Calavi (LHA/INE/UAC) \\ Philippe Laleye \\ Laboratoire d'hydrobiologie et d'Aquaculture, \\ Université d'Abomey-Calavi (LHA/FSA/UAC)
}

Doi:10.19044/esj.2021.v17n10p77

Submitted: 18 November 2020

Accepted: 12 February 2021

Published: 31 March 2021
Copyright 2021 Author(s)

Under Creative Commons BY-NC-ND

4.0 OPEN ACCESS

Cite As:

Yantikoua S., Laïbi R., Adechina R., Kaki C., Mama D. \& Laleye P. (2021). Dynamique hydrologique et évolution morpho sédimentaire du lac Ahémé au Sud-Ouest du Bénin. European Scientific Journal, ESJ, 17(10), 77. https://doi.org/10.19044/esj.2021.v17n10p77

\section{Résumé}

Le lac Ahémé dans le complexe estuarien sud-ouest béninois connait de nos jours, un véritable problème de dégradation dû à l'évolution naturelle et aux activités anthropiques ayant contribué à accélérer la vulnérabilité de l'hydrosystème. La présente étude, basée sur l'analyse de l'évolution bathymétrique et sédimentologique des fonds lagunaires vise à caractériser le rythme de comblement du lac Ahémé. La bathymétrie du lac Ahémé indique un plan d'eau encaissé, à fond plat, avec des profondeurs moyennes comprises 
entre 1,6 et 2,4 mètres. Les sédiments sont vaseux dans la partie centrale du Nord au centre du lac, sablo-vaseux au Sud et à l'Est puis sableux à l'Est et aux abords du lac en contact avec les plateaux de Terre de Barre. L'analyse comparative des cartes sédimentologiques et bathymétriques des années successives, a montré que le lac se comble au fil des ans, particulièrement dans sa partie sud. L'évolution des faciès sédimentaires du lac de 1999 à 2018 montre une plus large extension des vases et vases sableuses dans la partie sud du lac alors qu'elles se cantonnaient autrefois dans la moitié sud du lac. La répartition des vases n'est donc plus uniforme, comme c'était le cas il y a une vingtaine d'années. Une telle évolution bathymétrique est le signe tangible d'un comblement et cet état de chose est préjudiciable aux organismes vivants inféodés dans le milieu.

Mots clés : Lac Ahémé, sédimentologie, bathymétrie, comblement

\title{
Hydrological dynamics and morpho-sedimentary evolution of Lake Ahémé in South-West Benin
}

\author{
Sylvestre Yantikoua \\ Laboratoire de Géologie, Mines et Environnement, Département des \\ Sciences de la Terre, Université d'Abomey-Calavi \\ (LGME/DST/FAST/UAC). Ecole Doctoral Sciences de la Vie et de la Terre, \\ Université d'Abomey-Calavi (ED-SVT/FAST/UAC)

\section{Raoul Laïbi} \\ Rodrigue Adechina \\ Christophe Kaki \\ Laboratoire de Géologie, Mines et Environnement, \\ Département des Sciences de la Terre, Université d'Abomey-Calavi \\ (LGME/DST/FAST/UAC) \\ Daouda Mama \\ Laboratoire d'Hydrologie Appliquée, l'Institut Nationale de 1'Eau, \\ Université d'Abomey-Calavi (LHA/INE/UAC) \\ Philippe Laleye \\ Laboratoire d'hydrobiologie et d'Aquaculture, \\ Université d'Abomey-Calavi (LHA/FSA/UAC)
}

\begin{abstract}
Lake Ahémé in the south-western Beninese estuary complex is nowadays experiencing a real problem of degradation due to natural evolution and anthropic activities that have contributed to accelerate the vulnerability of the hydro system. The present study, based on the analysis of the bathymetric
\end{abstract}


and sedimentological evolution of the lagoon bottoms, aims at characterizing the filling rate of Lake Ahémé. The bathymetry of Lake Ahémé indicates an entrenched, flat-bottomed body of water with average depths between 1.6 and 2.4 meters. The sediments are muddy in the central northern part of the lake, sandy-muddy in the south and east, then sandy in the east, and on the edges of the lake in contact with the Terre de Barre plateaus. Comparative analysis of the sedimentological and bathymetric maps of successive years has shown that the lake fills up over the years, particularly in its southern part. The evolution of the sedimentary facies of the lake from 1999 to 2018 shows a wider extension of the mud and sandy mud in the southern part of the lake whereas they were formerly confined to the southern half of the lake. The distribution of mud is therefore no longer uniform, as was the case some twenty years ago. Such a bathymetric evolution is the tangible sign of a filling and this state of affairs is detrimental to the living organisms that are dependent on the environment.

Keywords: Lake Ahémé, sedimentology, bathymetry, infilling

\section{Introduction}

La zone littorale du Bénin dispose d'un écosystème aquatique, fourni et varié, représenté de l'aval vers l'amont par une composante maritime importante, un système de lagunes et de lacs et un réseau hydrographique dans lequel s'inscrit le lac Ahémé. Situé au Sud-Ouest du Bénin, le lac Ahémé est l'un des plans d'eau majeur du complexe fluvio-lagunaire sud-ouest et constitue le deuxième plan d'eau du Bénin en termes de superficie après le lac Nokoué à l'Est. Il bénéficie aussi bien des apports continentaux et marins. L'importance de sa biodiversité fait de lui un écosystème très attractif pour les populations. Mais, les pressions humaines et les contraintes naturelles auxquelles cet écosystème est aujourd'hui soumis à raison de sa position géographique, le fragilisent et rendent très vulnérable l'économie de la zone. En effet, le lac Ahémé est confronté à un véritable problème de comblement dû aux apports issus de l'érosion des plateaux le bordant, des bassins versants alimentant ses berges, et aux mauvaises pratiques de pêche contribuant à accélérer le processus de dégradation de l'environnement. Cette situation a eu pour conséquences, la réduction considérable des captures des ressources halieutiques, la migration des pêcheurs vers les autres pays de la sous-région à la recherche de zones de pêche plus favorables (Badahoui, 2008). L'objectif de cette étude, est d'évaluer le rythme de comblement du lac Ahémé face à la pression sans cesse croissante des activités anthropiques. Elle s'appuiera sur l'analyse des données bathymétrique et sédimentologique. La bathymétrie permettra de mettre en évidence la morphologie du fond du lac à l'échelle du 
temps. L'analyse sédimentologique rendra compte de l'hydrodynamique ce qui aboutira à la mise en relief de la répartition des différents facies.

\section{Présentation du milieu d'étude}

\subsection{Localisation et aspect géomorphologique}

La zone d'étude est située au Sud-Ouest du Bénin. Elle fait partie du complexe fluvio-lagunaire sud-ouest béninois qui regroupe le lac Ahémé, le chenal Aho et la lagune côtière, tous associés à un réseau très dense de chenaux de marée anastomosés souvent bordés de palétuviers. Le lac Ahémé est délimité par deux reliefs tabulaires inclinés en pente douce vers le Sud : les plateaux de Comé et d'Allada qui surplombent le lac avec des altitudes variant entre 40 et $50 \mathrm{~m}$. Les minces cordons sableux de la plage actuelle assurent la séparation entre le système lagunaire et l'océan atlantique (figures $1 \& 2$ ).

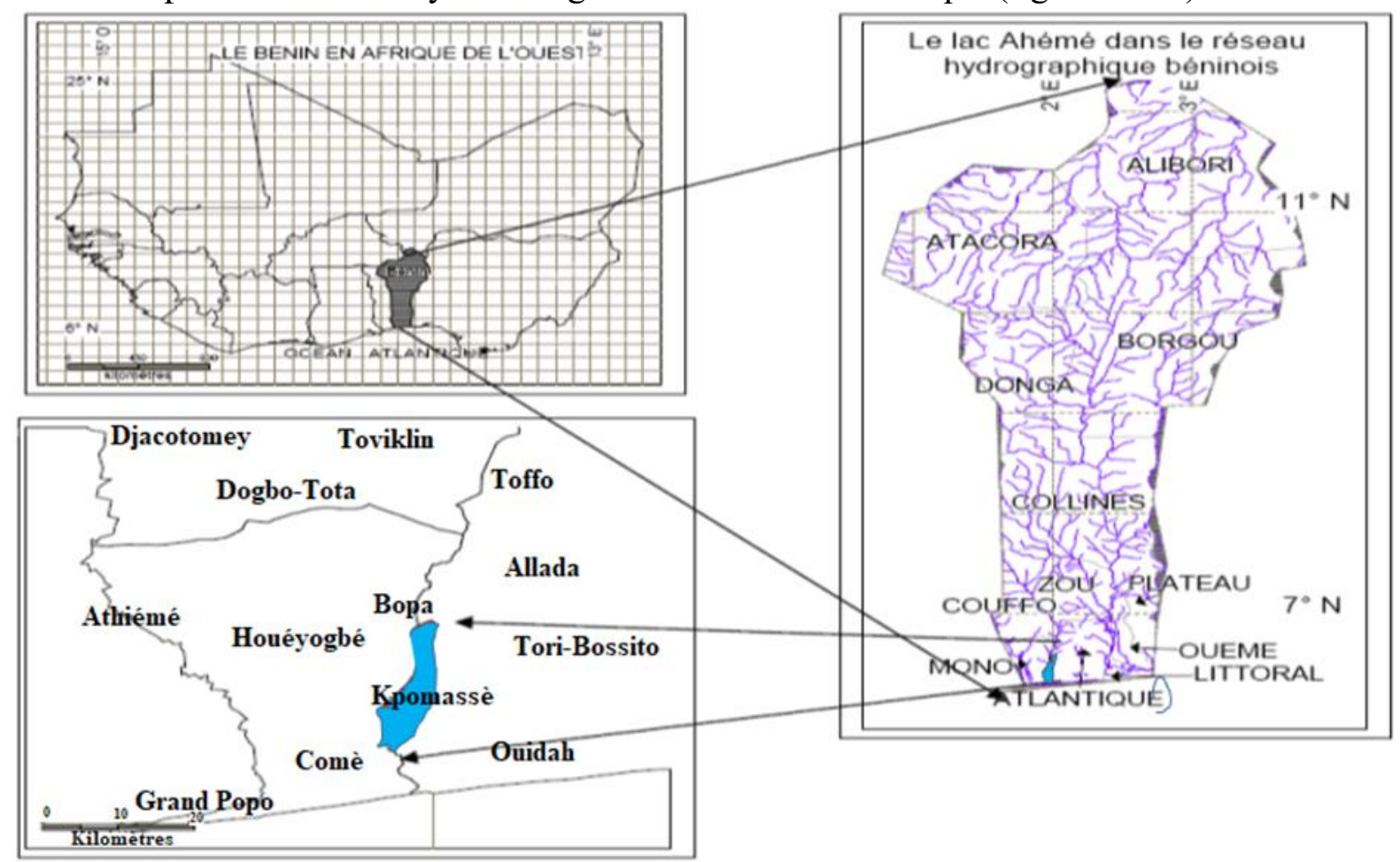

Figure 1 : Carte de localisation du lac Ahémé dans le littoral du sud-ouest béninois 


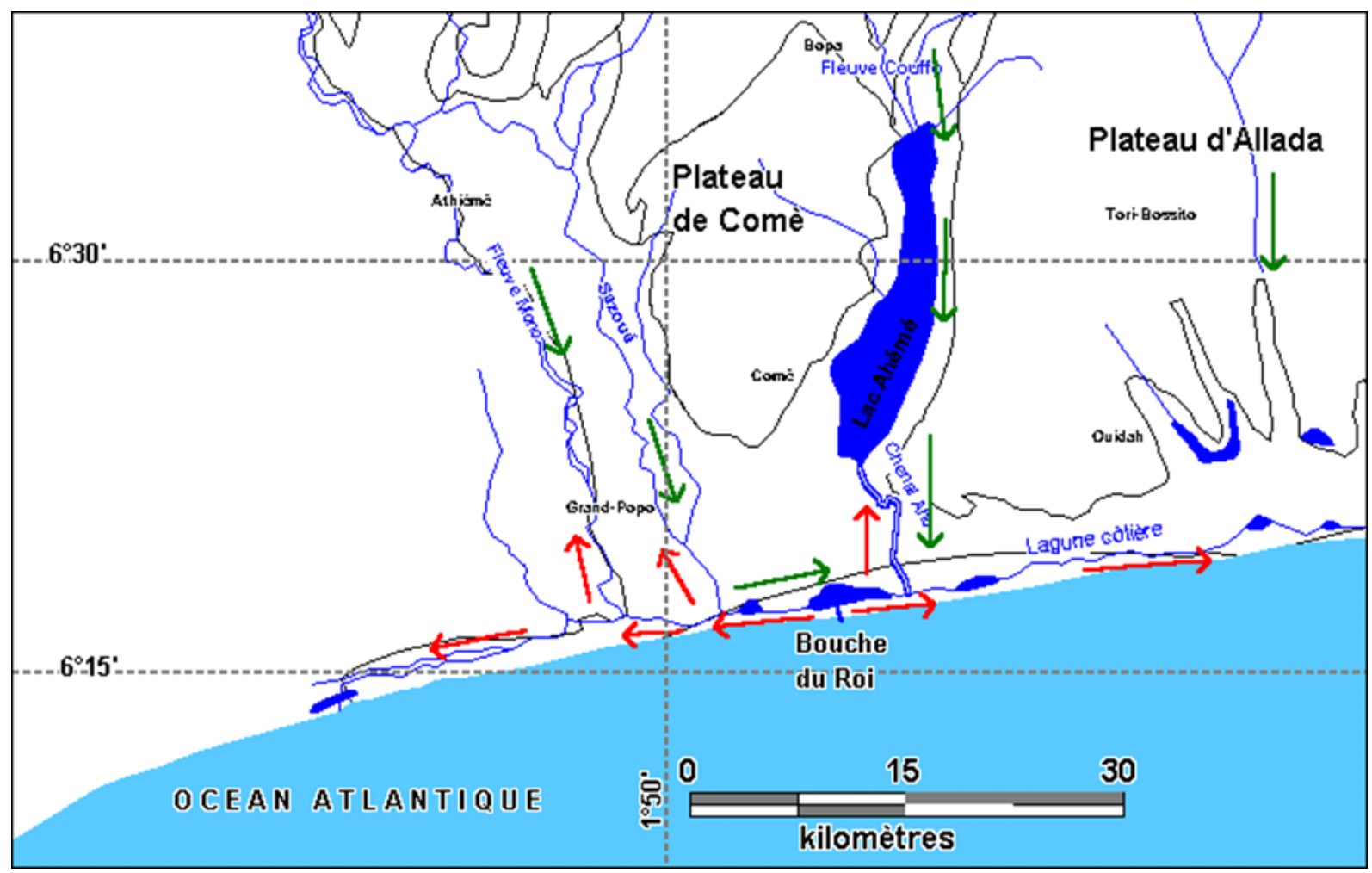

Figure 2 : Lac Ahémé dans le système hydrologique du complexe sud-ouest du Bénin Source : Base de données CATCH 2002

\subsection{Facteurs Climatiques}

Les principaux traits caractéristiques du climat dans le milieu d'étude sont ceux du Sud du Bénin, marqués par le climat subéquatorial de type bimodal avec quatre saisons dans l'année que sont : i) une grande saison des pluies de mi-mars à mi-juillet ; ii) une petite saison des pluies de mi-septembre à mi-novembre ; iii) une grande saison sèche de mi-novembre à mi-mars ; et iv) une petite saison sèche de mi-juillet à mi- septembre. La hauteur d'eau recueillie au cours de l'année varie entre 950 et $1300 \mathrm{~mm}$ (ASECNA, 2018).

De manière générale, les vents ont une orientation sud-ouest. Leur vitesse moyenne mensuelle varie entre 3 et $5 \mathrm{~m} . \mathrm{s}-1$. Les vents qui prédominent sur le littoral de Cotonou sont ceux du SW (64 \%) avec une forte fréquence dans les mois de février, mars, avril, juin et octobre. Les vents du WSW (16,07 $\%$ ), plus fréquents de juillet à septembre ont une vitesse moyenne mensuelle qui oscille autour de $6 \mathrm{~m} / \mathrm{s}$ (ASECNA, 2012). Ceux du SSW (14,40\%) sont très peu fréquents ces dernières décennies; leurs vitesses moyennes mensuelles décroissent de $5 \mathrm{~m} / \mathrm{s}$ en janvier à $3,6 \mathrm{~m} / \mathrm{s}$ en mars, novembre et décembre (ASECNA, 2012).

La température moyenne varie très peu dans le milieu littoral du Bénin (environ $27^{\circ} \mathrm{C}$ à Cotonou et à Ouidah). Les températures maximales sont 
relevées généralement au cours des mois de février à Avril, qui correspondent au pic de la saison sèche avec des journées ensoleillées $\left(31-33^{\circ} \mathrm{C}\right)$ suivies de nuit fraiches $\left(23-24^{\circ} \mathrm{C}\right)$. Les plus faibles s'observent entre Juillet et Août $\left(25^{\circ} \mathrm{C}\right)$.

\subsection{Hydrologie}

L'hydrologie continentale de la zone d'étude est marquée par le lac Ahémé, le Couffo qu'il reçoit en provenance du Nord, le chenal Aho qui le relie vers le Sud à l'océan atlantique par le biais de la lagune côtière qui le rattache au fleuve Mono.

D'une longueur de $24 \mathrm{~km}$ et d'une largeur variant entre 2 et $5,5 \mathrm{~km}$, le lac Ahémé a une superficie de $78 \mathrm{~km}^{2}$ à l'étiage et de $100 \mathrm{~km}^{2}$ en crue (Pliya, 1980). Ce plan d'eau, encaissé à l'intérieur des terres, présente des rives à parois plus ou moins abruptes et dissymétriques aux limites des plateaux de Terre de Barre. Les profondeurs inférieures sont à 1,5 $\mathrm{m}$ au Sud du lac, puis varient entre 1,5 et $2,5 \mathrm{~m}$ au centre et au Nord du lac. Toutefois, des profondeurs relativement importantes allant de 3 à $4,5 \mathrm{~m}$ peuvent être observées au Nord du lac à l'entrée du fleuve Couffo qui est son principal tributaire (Oyédé et al., 2007).

L'hydrologie marine qui joue un rôle important dans le fonctionnement du système lagunaire est caractérisée par la houle et la marée. Ces deux grands forçages océaniques contrôlent la dynamique du secteur d'une part, et influencent son système hydrologique, d'autre part. Les travaux de Guilcher (1959), Sitarz (1960), et Rossi (1989) montrent que la marée est du type semi-diurne avec des marnages extrêmes de $+1,95 \mathrm{~m}$ et $-0,20 \mathrm{~m}$, l'amplitude moyenne tournant généralement autour d'un mètre (type microtidal). La direction et le régime des houles sont liés à des tempêtes dans l'Atlantique sud, notamment, celles générées par l'anticyclone de Saint Hélène et, accessoirement, à des vents locaux. Elles font apparaître deux saisons de houle : l'une, avec des houles de faibles hauteurs $(0,4$ à $0,5 \mathrm{~m}$ en moyenne) d'octobre/novembre à mai ; l'autre, où, durant l'été boréal, de juin à septembre, les hauteurs atteignent et dépassent $2 \mathrm{~m}$. Les directions de houle sont constantes et montrent une prédominance des directions S à SSW pour les premières houles et SSW à SW pour les secondes. La houle est le facteur essentiel de la dynamique des sédiments essentiellement sableux sur le littoral béninois et a une période comprise entre $10 \mathrm{~s}$ et $15 \mathrm{~s}$ avec une fréquence moyenne de 11 - $12 \mathrm{~s}$. L'obliquité de la houle au déferlement par rapport au rivage varie entre $4^{\circ}$ et $9^{\circ}$, avec une moyenne autour de $6^{\circ}-7^{\circ}$. Elle entraîne un courant de dérive littorale dirigée d'Ouest en Est et dont la vitesse mesurée à Cotonou est de l'ordre de 0,3 à $1 \mathrm{~m} / \mathrm{s}$. Ce courant est responsable du transit de 1,2 à 1,5 million de $\mathrm{m}^{3}$ de sables de Lomé (à l'Ouest) à Cotonou (à l'Est) le long de la côte du golfe de Guinée, chaque année (Sitarz, 1960 ; NEDECO, 
1975 ; LACKNER, 1983 ; LCHF, 1984 ; Rossi et Antoine, 1990). La salinité des eaux marines varie de 34 à $36 \mathrm{~g} / \mathrm{L}$, la température de surface varie de $27^{\circ}$ à $30^{\circ} \mathrm{C}$ en saison chaude, et de $22^{\circ}$ à $26^{\circ}$ en saison froide (Abe, 1996).

\section{Matériel et méthode}

Les données traitées dans cette étude sont issues des campagnes bathymétrique et sédimentologique réalisées en Juillet-Août 2018. Le suivi des hauteurs d'eau aux stations d'Adjatokpa et de Bopa situées au bord du lac Ahémé a permis de calibrer les données par rapport à un niveau de référence. La campagne bathymétrique s'est déroulée en juillet-août 2018 à l'aide d'un traceur GPS MAP 521S équipé d'un sondeur à double fréquence suivant des transects de direction Est-Ouest distants en moyenne de $200 \mathrm{~m}$ l'un de l'autre.

\section{- Prélèvement de sédiment}

Le prélèvement de sédiment a été fait à la benne de Birg-Ekman (C'est un appareil qui sert à faire des prélèvements ponctuels de sédiments mous) suivant un quadrillage par des radiales est-ouest. La non-perturbation de l'échantillon à la remontée et la pénétration modérée dans la vase rendent cet échantillonneur très pratique pour l'étude des sédiments superficiels. Ensuite, ces échantillons ont été décrits sur place selon la caractérisation sédimentaire établie par Larsonneur en 1977 avant d'être emballé dans les sachets polyéthylène et étiquetés et transportés au laboratoire pour étude granulométrique. Les équidistances entre les radiales sont de $600 \mathrm{~m}$ dans le lac (Figure 3). Sur chaque radiale, les points d'échantillonnage ont été positionnés à des intervalles de $400 \mathrm{~m}$ (Figure 3 ). Les points d'échantillonnage ont été localisés le long des radiales à l'aide d'un GPS et localisés sur une carte topographique de l'IGN au 1/50 000. Aux différents points d'échantillonnages, le sens d'écoulement des eaux qui est tantôt dirigé vers le nord tantôt vers le sud a souvent apprécié.

\section{- Analyse granulométrique}

Elle est réalisée pour connaître la répartition quantitative de chaque classe dimensionnelle de particules élémentaires constituant l'échantillon. A cet effet, une masse $m t$ égale à $200 \mathrm{~g}$ de chaque échantillon de sédiments préalablement séchés a été prélevée et lavée sur un tamis à mailles carrées d'arêtes de $50 \mu \mathrm{m}$ pour séparer la fraction sableuse de la fraction siltoargileuse. Après séchage à l'étuve à une température de $80^{\circ} \mathrm{C}$ pendant 24 heures, la fraction sableuse recueillie est tamisée à sec sur une colonne de huit tamis (série AFNOR) de mailles d'arêtes décroissantes de $2 \mathrm{~mm}$ à $0,050 \mathrm{~mm}$. Ce tamisage est fait avec un appareil Rotap de type «Bioblock Scientific » pendant $10 \mathrm{mn}$ à une amplitude de 70 vibrations $/ \mathrm{mn}$. Chacune des fractions retenues par chaque tamis est pesée et consignée pour la construction 
d'histogrammes et de courbes cumulatives et le calcul des paramètres granulométriques de Folk et Ward (1957).

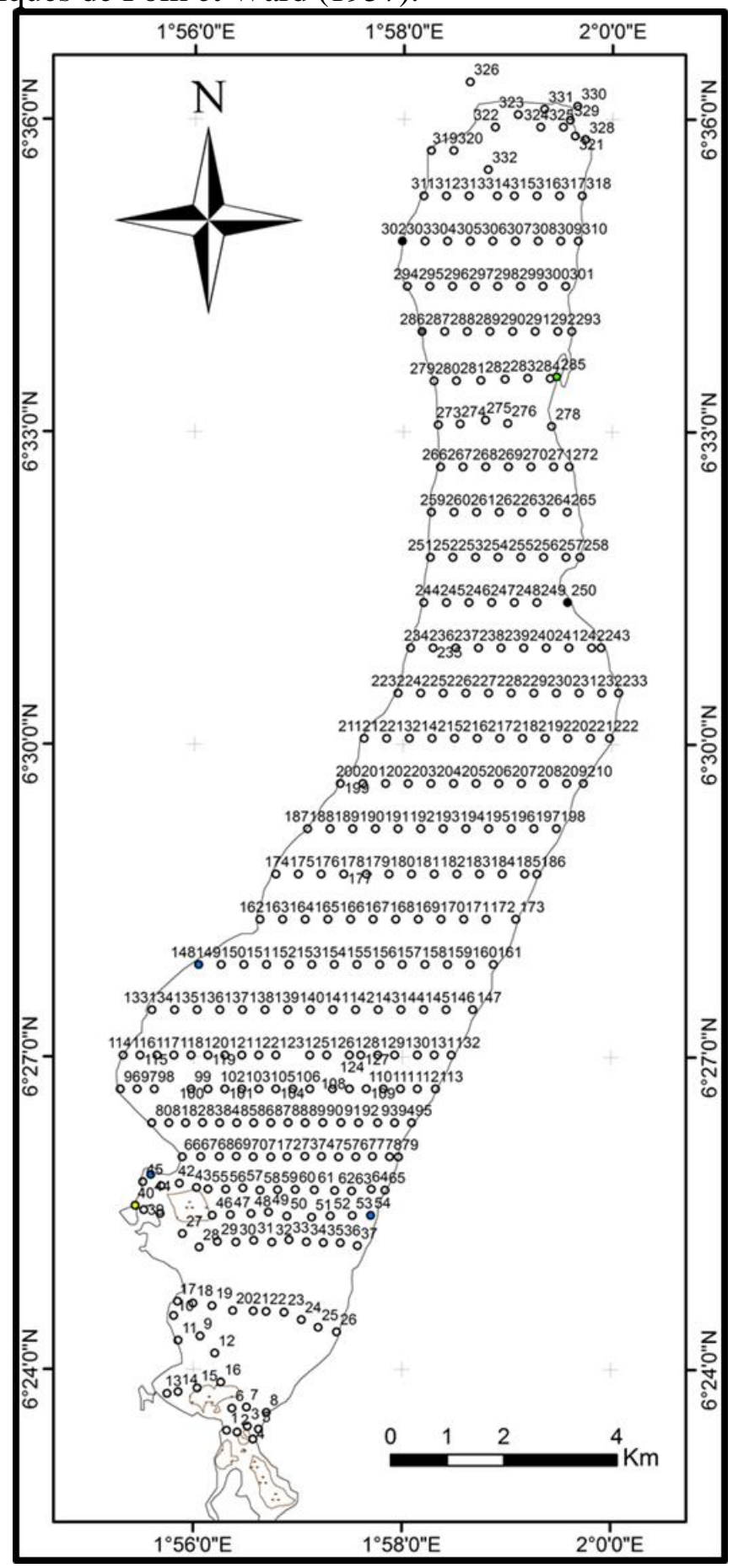

Figure 3: Position des sections bathymétriques et des points d'échantillonnage de sédiments dans le lac Ahémé 


\section{Résultats et discussion}

Pour décrire, les phénomènes de rétroaction entre hydrologie et sédimentologie au sein du lac Ahémé et ses chenaux, les études sédimentologiques et bathymétriques ont été actualisées dans le cadre de la présente étude. Les résultats obtenus ont été ensuite comparées aux données issues des travaux antérieurs. Faut-il le rappeler, le lac Ahémé constitue le centre d'intérêt du Département des Sciences de la Terre, depuis les années 1983 et bénéficie à ce titre d'une abondante revue bibliographique qui a permis de retracer l'évolution bathymétrique et sédimentaire avec notamment les travaux antérieurs de Oyédé, 1983 ; Montcho et Sègbédji, 1993 ; Roche International, 1999 ; SOMUSFOR, 2013, etc. C'est donc fort de ces connaissances étalées sur près de trois décennies que l'étude sur le comblement du lac Ahémé a été entreprise.

\subsection{Rappels sur le fonctionnement hydrologique du lac Ahémé avant et après la construction du barrage de Nangbéto sur le fleuve Mono}

Le lac Ahémé est un milieu complexe où arrivent les eaux du Couffo mais aussi du Mono et de la Sazoué ; notamment en saison du crue (Oyédé, 1983 ; Oyédé et al., 1988). Le chenal Aho d'orientation nord-sud relie le lac et la lagune. Chaque année, la première crue de juin-juillet du Couffo est très déterminante sur la montée du niveau des eaux du système qui demeurent toutefois saumâtres. Par contre, en août-octobre, (crue du Mono), que ce soit avant ou après la mise en service du barrage, les courants du Mono deviennent très forts dans le système lagunaire et les eaux y deviennent purement douces. Ainsi, à basse marée, la quasi-totalité des eaux du Mono traverse la lagune côtière et se déverse directement dans la mer par la Bouche du Roy (Oyédé, 1983). A haute marée, bien que l'essentiel des eaux du Mono se déversent aussi dans la mer par la Bouche du Roy, une partie remonte le chenal Ahô pour se stocker dans le lac Ahémé (Pliya, 1976 ; Oyédé, 1983). Durant l'étiage du Mono, avant la mise en service du barrage, du fait qu'aucun apport d'eau douce substantiel n'arrive dans l'estuaire, les échanges hydrologiques s'effectuent quasi exclusivement avec les eaux marines et la salinité dans l'estuaire reste élevée (Oyédé, 1983 ; PNUD, 1987). Après le barrage, sous l'effet de la permanence des écoulements du fleuve Mono et de l'importance de leurs débits, les équilibres d'eau dans l'estuaire s'effectuent désormais avec un mélange eaux marines - eaux du Mono. Ainsi, le régime d'eau purement marine de l'estuaire en saison sèche avant le barrage fait place à un régime d'eaux saumâtres après le barrage (Oyédé, 1983).

Par ailleurs, après la mise en eau du barrage de Nangbéto, l'installation d'un écoulement fluvial d'étiage permanent et non négligeable fait que le 
niveau d'eau dans le système est désormais plus élevé à l'étiage qu'auparavant (on passe de $0,09 \mathrm{~m}^{3} / \mathrm{s}$ à $39 \mathrm{~m}^{3} / \mathrm{s}$ ).

En conclusion, avant comme après le barrage de Nangbéto, le système lagunaire voit ses eaux s'écouler dans un sens ou dans l'autre en fonction des marées et des saisons hydrologiques des fleuves Mono et Couffo. La salinité des eaux de l'estuaire varie également en fonction des coefficients de marée et des saisons hydrologiques des fleuves Mono et Couffo.

\subsection{Caractéristiques morphologiques du lac Ahémé}

\subsubsection{Analyse bathymétrique}

La carte bathymétrique est le reflet de la disposition des sédiments au fond du lac. C'est elle qui traduit l'évolution morphologique entre les mesures de deux périodes différentes. Les mesures effectuées dans le cadre de cette étude ont permis d'établir la carte bathymétrique de la Figure 4.

\subsubsection{Description des fonds bathymétriques de la campagne de juillet-août 2018}

L'analyse de la Figure 4 montre que, du point de vue de la bathymétrie des fonds, le lac présente trois domaines :

- Les fonds entre 0,4 et 1,6 mètre de profondeur

Ils correspondent à la bordure du lac, avec une dénivellation de 1,2 mètre. Une telle dénivellation de bordure confirme que le lac Ahémé est un plan d'eau lagunaire encaissé. Toutefois, la pente est beaucoup plus douce dans la partie sud-ouest du lac, entre Akodéha et Guézin.

- Les fonds entre 1,6 et 2,4 mètres de profondeur

Ils représentent la grande partie de l'étendue du lac, laissant apparaître une configuration plate du fond du lac.

- Les fonds entre 2,4 et 3 mètres de profondeur

Ils forment les plus importantes profondeurs du lac, définissant un domaine plus étroit structuré sous forme de cuvettes localisées dans la partie nord orientale du lac, entre Tokpa Domè et Dékanmè.

\subsubsection{Evolution bathymétrique du lac Ahémé}

Des relevés bathymétriques ont été faits en 1983, 1991, 1999 et en 2013. La comparaison de relevés actuels avec les précédentes mesures montre qu'il se produit constamment un remaniement dans le fond du lac, d'une année à une autre.

En comparant les anciens relevés de 1999 et de 2013 aux données de cette dernière campagne, nous observons dans la partie sud du lac, un fond qui ne présente plus une large cuvette comme en 2013, mais plutôt une configuration globalement en forme de «U $\mathrm{U}$ comme en 1999 (Figure 5). Cette 
disposition du fond du lac dans sa partie sud laisse comprendre pourquoi les vases et vases sableuses tendent à recouvrir le fond du lac qui devient alors beaucoup plus plat. Une telle évolution a été de même observée dans les chenaux de la lagune de Fresco en Côte d'Ivoire par N'guéssan, (2013). Cette configuration estime-t-il, traduirait un équilibre d'action entre les agents d'érosion et les agents d'accumulation. Cette évolution bathymétrique est le signe tangible d'un comblement et cet état de chose est préjudiciable aux organismes vivants inféodés dans le milieu.

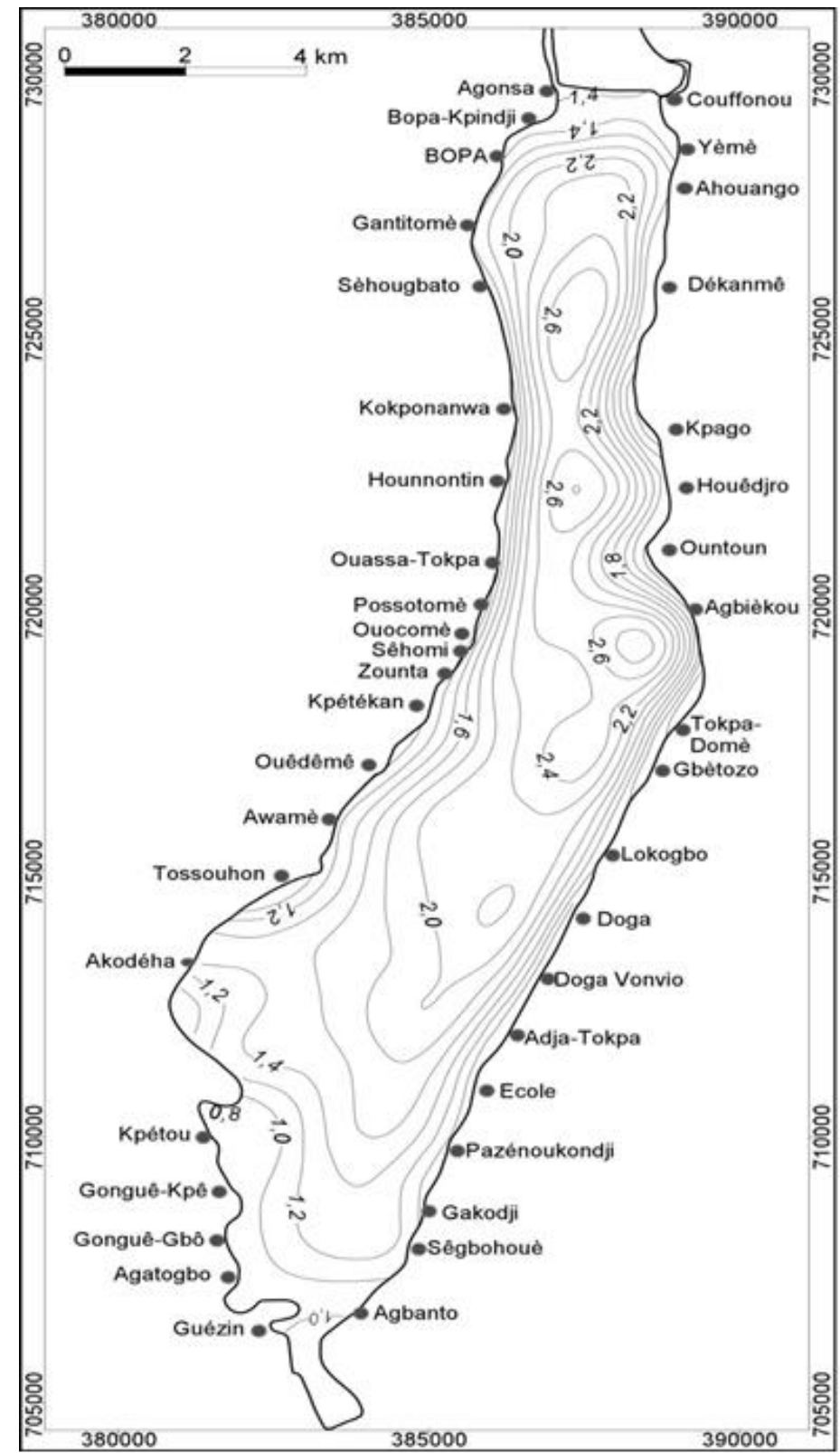

Figure 4: Carte bathymétrique du lac Ahémé (juillet-août 2018) 


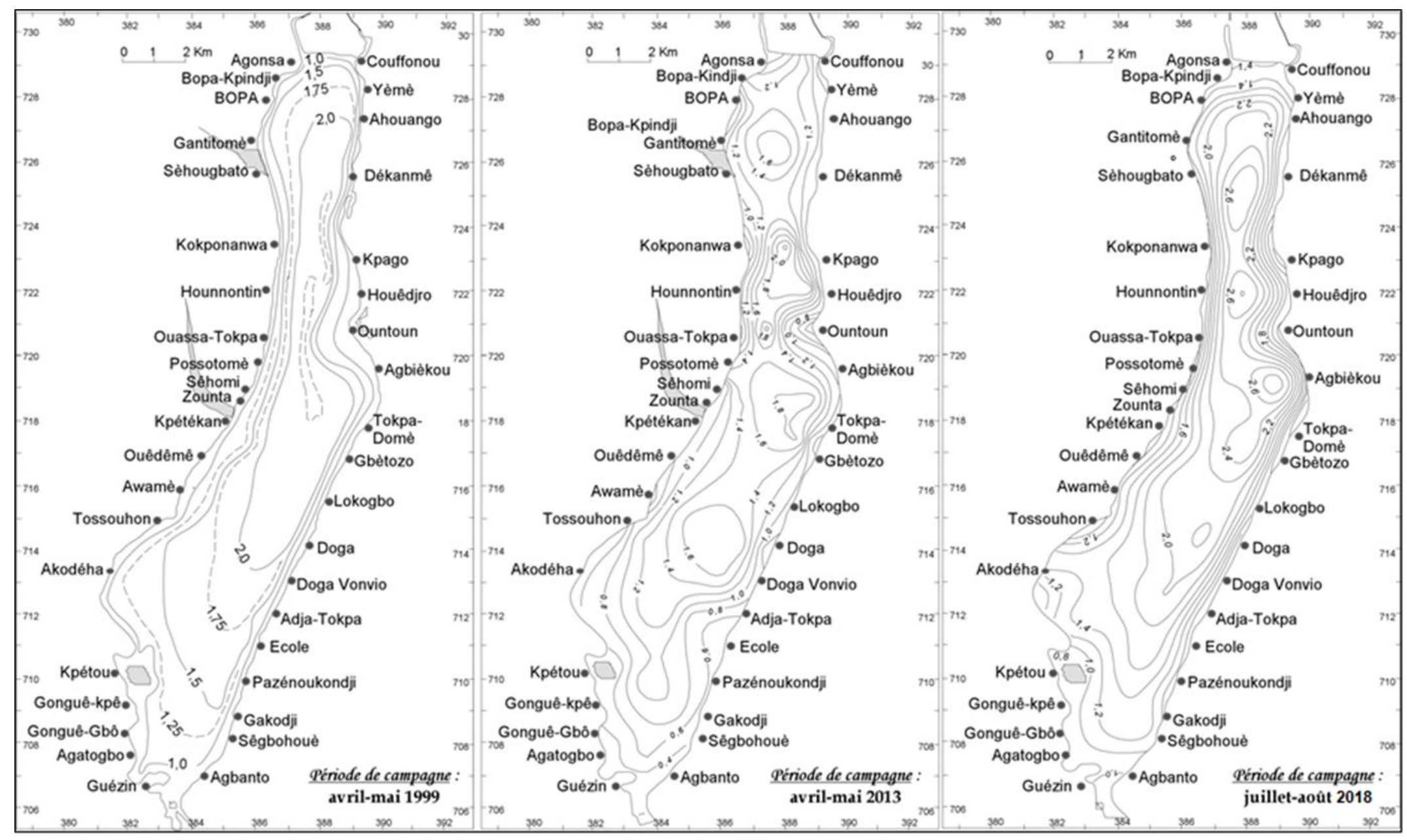

Figure 5: Cartes bathymétriques des années 1999, 2013 et 2018 


\subsection{Répartition des faciès sédimentologique du lac Ahémé}

Analyse des lithofaciès du fond du lac Ahémé et ses chenaux

Les sédiments de fond du lac Ahémé décrits dans le cadre de cette étude sont repartis en quatre faciès types (Figure 6) :

- le faciès de sables : Il se rencontre en bordure du lac au pied des plateaux du Continental terminal et de la Terre de barre. Mais, il est particulièrement développé sur la rive est et surtout sur la moitié nordest du lac où son étendue peut dépasser le kilomètre de large, entre Agbékou, Hountoun, Houédjro et Kpago. Il s'agit des sables fins à moyens et des sables moyens, très peu coquilliers et assez propres. Il s'accumule à la faveur des clapots (ou vagues) liés au vent du SudOuest et qui battent, à l'image des vagues en mer, la rive Est du lac.

- le faciès de sables vaseux : Son extension est limitée au Sud du lac. Il s'agit essentiellement des sables fins à moyens, souvent coquilliers.

- le faciès de vases sableuses : Il est présent dans tout le lac où il se retrouve autour du faciès sableux en progressant des rives vers l'intérieur du lac.

- le faciès de vases : les vases occupent largement la partie centrale du lac où elles montrent une extension nord-sud, avec une abondance particulière dans la partie sud du lac Au nord, dans le prolongement des débouchés du Couffo dans le lac, les sédiments vaseux ont également une large extension ; ce qui montre que le Couffo est pauvre en charges sédimentaires sableux. 


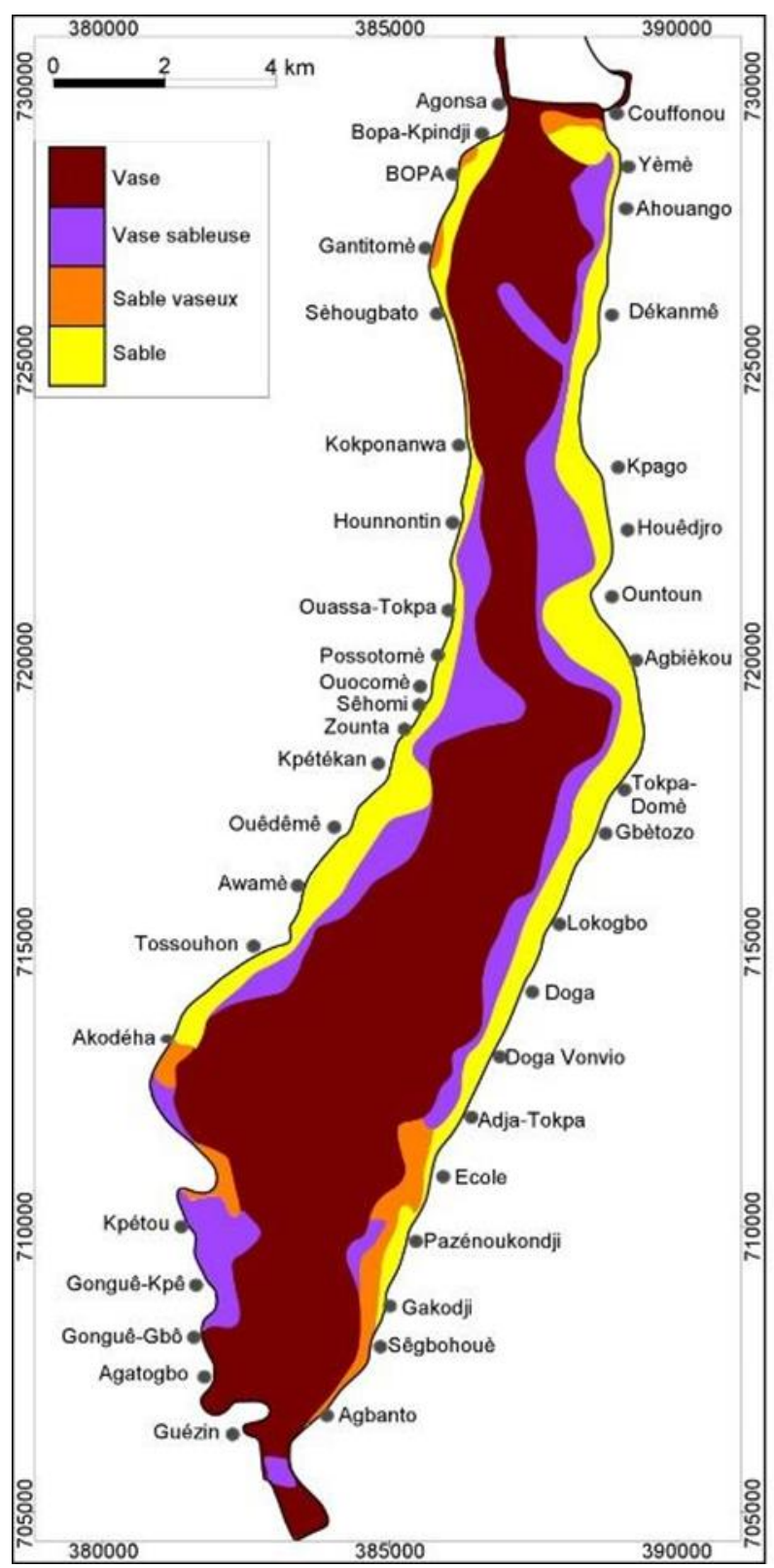

Figure 6 : Carte des lithofaciès du lac Ahémé

\subsubsection{Evolution sédimentologique et morphologique du lac Ahémé}

Essai de comparaison des lithofaciès actuels du lac avec ceux des années antérieures

Les faciès du lac Ahémé ayant été suivis depuis 1981, une évolution significative peut être notée dans la répartition des sédiments du fond du lac. En effet, d'après les travaux de Oyédé (1983), le lac se comportait en première approximation comme un bassin de sédimentation où les faciès de bordure sont 
plus ou moins sableux, tandis que les particules fines sont transportées au centre du lac.

Nous avons limité la comparaison des résultats actuels aux deux dernières cartes de 1999 et de 2013 (Roche International, 1999 et Somusfor, 2014). En effet, par rapport aux cartes de faciès des années 1999 et 2013, nous notons avec la campagne de 2018, une plus large extension des vases et vases sableuses qui tendent à tapisser tout le fond du lac (figure 7). Les vases et vases sableuses gagnent de plus en plus la partie nord du lac, alors qu'elles ne se limitaient seulement que dans la moitié sud du lac en 1999. La répartition des vases n'est donc plus uniforme, comme c'était le cas il y a une quinzaine d'années. Dans la partie nord du lac, les vases et vases sableuses tendent à recouvrir les sables vaseux qui servaient de transition entre les sables francs et les vases sableuses, notamment entre Yèmè et Dékanmè d'une part, et entre Agonsa et Sèhougbato d'autre part.

Dans la moitié nord de la rive est, l'accumulation du sable est permanente entre Agblékou et Kpago, en liaison avec les vents locaux. 

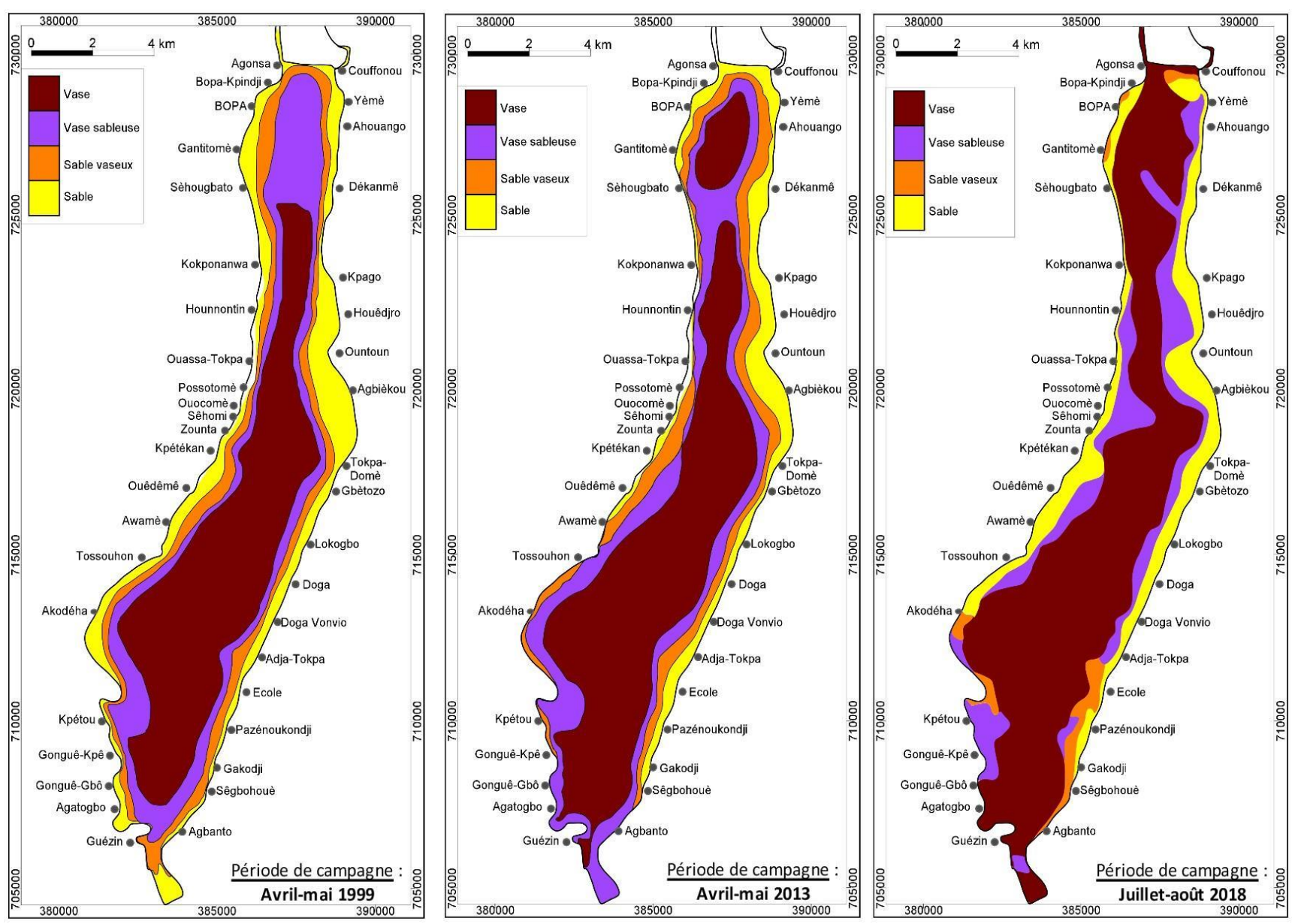

Figure 7: Cartes sédimentologiques du lac Ahémé des années 1999, 2013 et 2018 


\subsection{Distribution granulométrique des sédiments du lac Ahémé}

L'étude granulométrique des échantillons sableux du lac Ahémé a permis de construire les courbes cumulatives afin de calculer les paramètres granulométriques. Ces paramètres permettent de caractériser les sédiments en remontant aux conditions de leur dépôt.

Les courbes cumulatives des faciès du lac Ahémé (Figure 8) présentent pour la plupart une allure logarithmique (avec une faible pente) et d'autres ont une tendance hyperbolique et quelques rares courbes ont une tendance parabolique.

Ces faciès sableux présentent un indice d'asymétrie compris entre $0,05 \Phi$ et $0,17 \Phi$ qui est tantôt symétrique vers les fractions fine ou asymétrique vers les fractions grossières. L'écart-type oscille entre $0,84 \Phi$ et $1,08 \Phi$, ce qui indique des particules médiocres à moyennement triés. Ces différents paramètres calculés, montrent que le processus de sédimentation dans le lac résulterait de l'influence de plusieurs sources d'apports sédimentaires présentant des courants de différentes intensités. Or, le lac Ahémé de par sa position géomorphologique, a la particularité d'être alimenté à la fois par les eaux marines et continentales et de ce fait, reçoit en période de crue des matériaux fluvuo-lagunaires. La prépondérance des courbes logarithmiques montre que le pourcentage des particules fines $(<0,063 \mathrm{~mm})$ laisse penser à un ralentissement de l'énergie de transport de sédiments. Cette hypothèse semble conforme aux travaux de Guilcher (1959) et Pliya (1980) qui, abordant l'étude du phénomène de comblement du lac Ahémé, ont pu mettre en évidence la présence des flèches qui barrent transversallement les plans d'eau occasionnant ainsi le piégeage des éléments remaniés par la végétation environnante. Ces auteurs ont aussi fait remarquer que le comblement du lac s'expliquerait non seulement par un phénomène naturelle inéluctable lié à la sédimentation et à l'alluvionnement annuelle des crues du Couffo et du Mono mais également par l'érosion des bassins versants et à la destruction des palétuviers fixateurs de la vase. La nouvelle carte sédimentologique (2018) montre que la partie nord du lac proche du fleuve Couffo est plus enrichie davantage en vase qu'en 2013. Or, selon les travaux de Castings (2008), en période de crue alors que le courant est plus fort, ce sont des particules fines qui sont transportées en majorité. Mieux, l'inondation des terres et le ruissellement abondant, poursuit-il, chargent massivement le cours d'eau en particules terrigènes argileuses. Cette observation permet donc de comprendre que l'enrichissement en vase dans la partie nord (zone bordière du fleuve Couffo) et au sud du lac, est dû aux crues des fleuves Couffo et Mono. En d'autres termes, en période de crue, le lac voit les eaux du fleuve Couffo se mélanger avec les eaux de la lagune côtière (salée) par le truchement du chenal Aho. Ce mélange alors chargé de particules fines silto argileuses provoque une floculation importante de particules en suspension qui se 
déposent par excès de charge et explique l'envasement généralisé observé au sud du lac au déboucher du chenal Aho notamment à Guézin, Agatogbo et Conguè-Gbo.

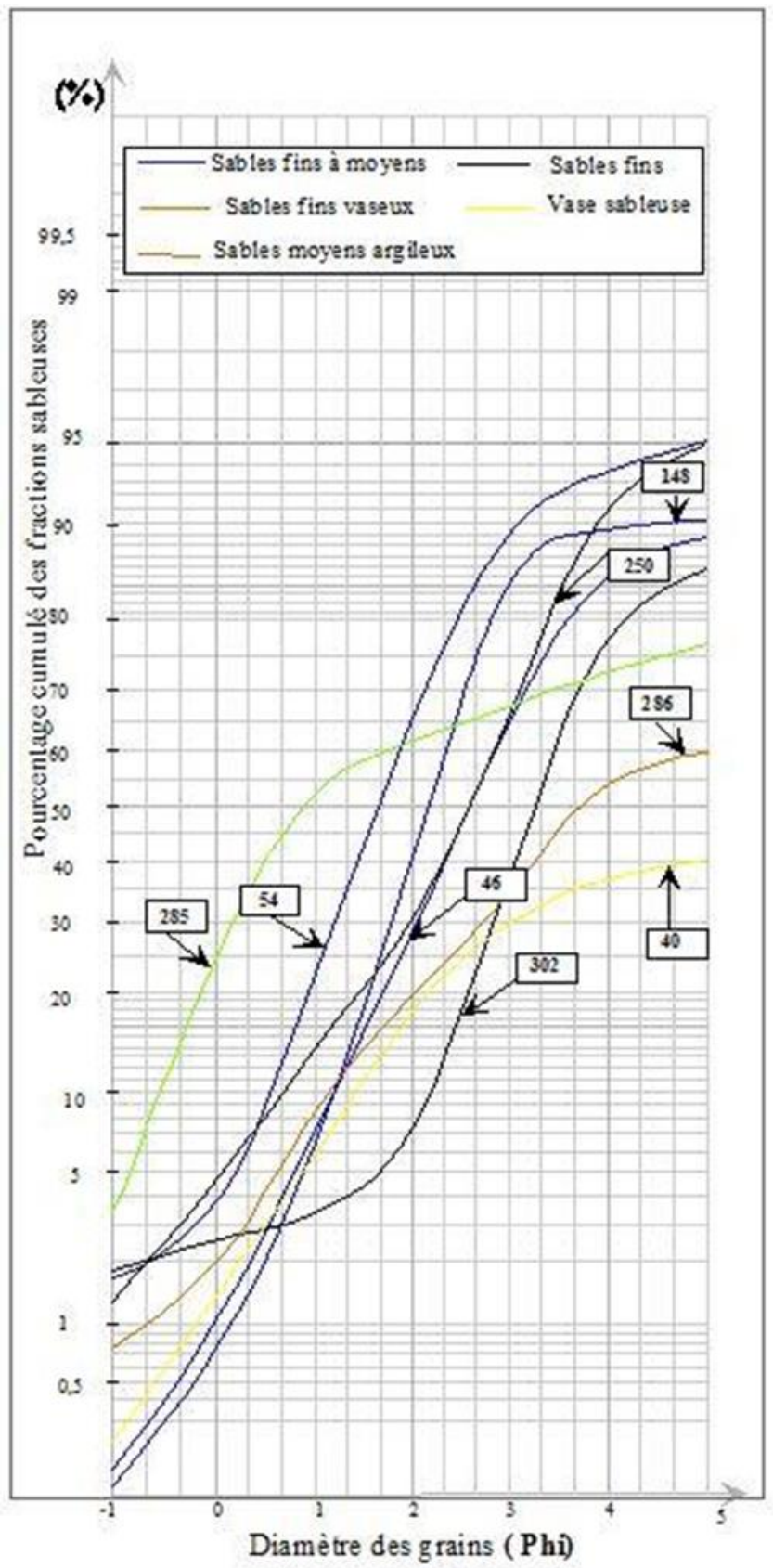

Figure 8: Courbes cumulatives des différents faciès sédimentaires dans le lac Ahémé 


\subsection{Analyse des faciès vaseux du lac Ahémé : évaluation des teneurs en matière organique (MO)}

Les vases occupent l'axe central du lac Ahémé. Les pourcentages de sables sont inférieurs à $25 \%$; ce qui ne permet pas d'évaluer les indices granulométriques.

Par contre, les teneurs en matière organique (MO) ont été déterminées par la méthode de la perte au feu. Les résultats montrent que les teneurs en MO des sédiments superficiels de l'axe central du lac Ahémé varient de 2,27 $\%$ à $12,5 \%$ avec une moyenne de 5,87\% (Figure 7). Les sédiments analysés présentent majoritairement de fortes teneurs en $\mathrm{MO}$, car les valeurs excèdent largement $4 \%$ (entre $4 \%$ et 10\%). Par ailleurs, les teneurs en MO comprises entre 2 et $3 \%$ dénotent que le lac est moyennement pourvu en matière organique. De telles teneurs s'observent au Sud-Ouest du lac; alors que les secteurs à teneurs en $\mathrm{MO}$ comprises entre 3 et $4 \%$ caractérisent les parties sud-ouest et sud-est du lac bien pourvus en MO (Figure 8). La teneur maximale en MO du sédiment (12,5\%) est observée au Nord du lac.

\subsection{Facteurs de comblement du lac}

Par son cadre géomorphologique, le lac Ahémé constitue un environnement sédimentaire original, qui illustre cependant, les phénomènes hydrologiques et sédimentologiques intervenant dans un milieu de mangrove en climat tropical humide. L'héritage à partir du continent est dominant. Les études antérieures ont montré que l'essentiel des matériaux détritiques (sables et argiles) du lac proviennent des plateaux de ses bordures. En effet, le lac Ahémé est très encaissé et les pentes souvent abruptes des plateaux favorisent l'érosion de ses derniers par les eaux de ruissellement à la faveur des zones dénudées (pratiques agricoles, agglomérations avec des toitures en tôle, nombreuses pistes menant dans ces agglomérations). Les nombreux ravins en bordure des pistes et le déchaussement des cocotiers et autres arbres observés dans les localités en sont très illustratifs (Oyédé et al., 2007, Amoussou et al., 2015, Amoussou et al., 2016).

Bien que la problématique du comblement des milieux lagunaires s'inscrive dans l'évolution naturelle de tout plan d'eau plus ou moins fermé, il convient cependant de préciser que ce phénomène peut être aussi accéléré et/ou accentué par les activités anthropiques. Ainsi, les facteurs qui contribuent à la dégradation et au comblement des plans d'eau souvent liés à l'homme sont nombreux. Les techniques de pêche, notamment les Xha, les Acadja, les parcs à poisson freinent les courants d'eau en favorisant le dépôt des vases. Il y a également l'action des végétaux et des animaux qui enrichissent le milieu en matière organique.

Par ailleurs, les études sédimentologiques ont révélé que les apports du bassin versant du fleuve Mono et les apports d'origine marine prédominent 
dans le lac (Oyédé 1983 ; Oyédé et al., 2007 ; Roches International 2000 ; LCHF, 1987 ). Par contre, les apports sédimentaires du Couffo sont très insignifiants (Oyédé, 1983).

$\mathrm{Au}$ total, la construction des pistes et des agglomérations autour du lac; certaines techniques de pêche; les pratiques agricoles, le déversement des ordures dans le lac, l'érection du barrage de Nangbéto sur le fleuve Mono (Oyédé et al., 2007 ; Badahoui 2010) sont les principaux facteurs anthropiques favorisant le comblement du lac Ahémé.

\section{Conclusion}

L'organisation sédimentaire dans le lac Ahémé montre des vases et vases sableuses dans l'axe central du plan d'eau, les sables étant localisés dans les bordures du lac. Les sables sont à dominance constitués de grains fins à moyens, tandis que les vases sont très riches en matière organique. Les fonds bathymétriques du lac Ahémé montrent un plan d'eau encaissé, à fond plat, avec des profondeurs moyennes comprises entre 1,6 et 2,4 mètres. L'analyse comparative des cartes sédimentologiques et bathymétriques des années successives, a montré que le lac se comble au fil des ans, particulièrement dans sa partie sud. Les très fortes teneurs en matière organique dénotent par ailleurs d'un état anoxique avancé du lac dû à une augmentation excessive de la demande en oxygène ; ce qui aggrave l'état d'envasement du lac. Il importe donc de veiller sur ce plan d'eau à travers un schéma directeur d'aménagement qui garantit une bonne maîtrise de l'hydrodynamisme des écoulements, gage du maintien de la santé hydrologique du lac.

Enfin, l'étude sédimentologique et bathymétrique montre que le régime hydrologique du lac Ahémé contrôle la dynamique sédimentaire du système. L'interaction entre régime hydrologique et dynamique sédimentaire définit une boucle de rétroaction morphodynamique qui se traduit par des phénomènes de comblement et de creusement, lesquels déterminent la variabilité de la bathymétrie des fonds.

Que le phénomène soit lié à une évolution naturelle ou aux activités humaines, les conséquences écologiques et économiques de ces modifications sont souvent considérables. De même, les processus entrant en jeu dans le comblement sédimentaire sont complexes et variés. C'est pourquoi, leur prise en compte à travers une vision pluridisciplinaire de gestion de ce plan d'eau, s'avère nécessaire afin de mieux cerner le phénomène et mettre en place des mesures durables qui limiteraient les impacts sur les sociétés.

\section{References :}

1. Abe, J., Bakayoro S., Bamba, S. et Cissoko, S. (1996). L'hydrologie de l'estuaire du Comoé à Grand Bassam (Côte d'Ivoire). Agronomie 
Africaine. Journal. Ivoirien d'Océananographie .et Limnologie. 8(3) 201-212.

2. Amoussou E., Totin H., Kaki, C., Houessou S., Houndénou C., Gil Mahe, Oyédé, L.M. et Blivi B. (2015). Evolution sédimentologique et dynamique des charges solides dans l'hydrosystème fluvio-lagunaire du sud-ouest du Bénin. Conférence Internationale sur l'Hydrologie des Grands Bassins Fluviaux de l'Afrique, Oct 2015, Hammamet, Tunisie. /hal-02146325.

3. Amoussou, E., Kaki, C., Vissin W. E., Bamisso, R., Oyédé, L.M. (2016). Evolution sédimentaire et mutation des écosytèmes du lac Ahémé au sud-ouest. 7 (11) 192-203.

4. Badahoui, A., Fiogbe, E. D., Boko, M. (2010). Les causes de la dégradation du lac Ahémé et ses chenaux. Intérnational. Journal. Biological. Chemical. Sciences, 4, 882-897.

5. Castings, J. (2008). État de l'art des connaissances du phénomène de comblement des milieux lagunaires. Rapport de phase 1 Réseau de Suivi Lagunaire.pp100.

6. Laibi R. Hounkpe, J. B., Kelome, N. C., et Oyédé, L. M. (2017). Evolution morpho-sédimentaire du lac Ahémé dans la zone margino littoral du Bénin, Annales des Sciences. Agronomiques du Bénin. 21(2), 247-261

7. Guilcher, A. (1959). La region du Bas-Dahomey occidental. Etude de géographie physique et humaine appliquée. Institut Fondamental d'Afrique Noire T. XXI, Sér. B, n³-4, pp 358-424Lackner \& Partners (1983). Etude de l'ensablement de l'accès au port de Lomé. Rapport d'étude, Bremen, Ministère de l'Equipement, Lomé

8. LCHF (1984). Projet Erosion Littorale du Togo. Rapport d'étude et propositions d'aménagement, Université de Lomé, 80 p.

9. NEDECO. (1975). Erosion littorale sur la côte togolaise. Rapport d'étude. Ministère des travaux Publics, Lomé, 73 p.

10. Oyédé, L. M. (1983). Un exemple de sédimentation bio détritique quaternaire dans le domaine margino-littoral en climat tropical humide : le lac Ahémé (Bénin-Afrique de l'Ouest). Thèse de doctorat de $3^{\mathrm{e}}$ cycle en géologie sédimentaire de 1'Université de Dijon, $171 \mathrm{p}$.

11. Oyédé, L. M., Kaki, C., et Laibi, R. (2007). Environnement sédimentaire, morphologie et faciès du lac Ahémé dans le complexe lagunaire sud-ouest béninois. Annales des Sciences. Agronomiques du Bénin 9 (1), 75 - 98.

12. Pliya, J. (1980). La pêche dans le sud-ouest du bénin. Etude de géographie appliquée sur la pêche continentale et maritime. Paris : Acct, $296 \mathrm{p}$. 
13. Roche International. (2000). Etude d'aménagement des plans d'eau du Sud Bénin : hydrologie et sédimentologie. Rapport préliminaire, $87 \mathrm{p}$.

14. Rossi, G. (1989). L'érosion du littoral dans le Golfe du Bénin : un exemple de perturbation d'un équilibre morphodynamique. Zeitschrift für Geomorphologie: Supplementbände, 73,139-165.

15. Rossi, R., \& Antoine., P. (1990). Impacts hydrologiques et sédimentologiques d'un grand barrage: l'exemple de Nangbéto (Togo-Bénin). Revue de de Géomorphologie, T XXXIX N²-1990.

16. Sitarz, J.A. (1960). Côtes africaines : Etude des profils d'équilibre de plage. Trav. Centre d'Etude et de Recherche en Océanographie, T3, fasc. 4, pp.43-62.

17. SOMUSFOR. (2014). Étude de faisabilité du programme intercommunal de réhabilitation du complexe fluvio-lacustre du lac Ahémé et ses chenaux et de mise en place d'une zone de développement économique. Rapport Vol1 : Document principal, PIRA, Cotonou, $207 \mathrm{p}$.

18. N'guessan, A.Y., Adopo, K.L., Amani, M.E., Konan, K.B., Toure, M., Monde, S. et Aka, K. (2013). Etude bathymétrique, sédimentologique et environnement de dépôts des sables superficiels de la lagune de Fresco (zone ouest du littoral ivoirien). Journal of Asian Scientific Research, 2013, 3 (3)308-320.

19. N'guessan, A.Y., Wango, T.E., Konan, K.E., Adingra, A., Amani, M.E., Monde, S., Afian, K. et Aka, K. (2015). Hydrologie et morphologie de l'estuaire du fleuve Sassandra, Basse Côte d'Ivoire. Afrique SCIENCE 11(2) 161-172 